\title{
Holographic 3D tracking of microscopic tools
}

\author{
Glückstad, Jesper; Villangca, Mark Jayson; Bañas, Andrew Rafael; Palima, Darwin
}

\section{Published in:}

Proceedings of SPIE

Link to article, DOI:

$10.1117 / 12.2176801$

Publication date:

2015

Document Version

Publisher's PDF, also known as Version of record

Link back to DTU Orbit

Citation (APA):

Glückstad, J., Villangca, M. J., Bañas, A. R., \& Palima, D. (2015). Holographic 3D tracking of microscopic tools. In Proceedings of SPIE: Optical Pattern Recognition XXVI (Vol. 9477). [947702] SPIE - International Society for Optical Engineering. Proceedings of SPIE - The International Society for Optical Engineering https://doi.org/10.1117/12.2176801

\section{General rights}

Copyright and moral rights for the publications made accessible in the public portal are retained by the authors and/or other copyright owners and it is a condition of accessing publications that users recognise and abide by the legal requirements associated with these rights.

- Users may download and print one copy of any publication from the public portal for the purpose of private study or research.

- You may not further distribute the material or use it for any profit-making activity or commercial gain

- You may freely distribute the URL identifying the publication in the public portal 


\title{
Holographic 3D tracking of microscopic tools
}

\author{
Jesper Glückstad*, Mark Villangca, Andrew Bañas, Darwin Palima \\ DTU Fotonik \\ Programmable Phase Optics \\ Technical University of Denmark \\ DK-2800 Kgs. Lyngby, Denmark \\ *Email: jesper.gluckstad@,fotonik.dtu.dk \\ www.ppo.dk
}

\begin{abstract}
We originally proposed and experimentally demonstrated the targeted-light delivery capability of so-called Wave-guided Optical Waveguides (WOWs) three years ago. As these WOWs are maneuvered in 3D space, it is important to maintain efficient light coupling through their integrated waveguide structures. In this work we demonstrate the use of real-time diffractive techniques to create focal spots that can dynamically track and couple to the WOWs during operation in a volume. This is done by using a phase-only spatial light modulator to encode the needed diffractive phase patterns to generate a plurality of dynamic coupling spots. In addition, we include our proprietary GPC Light Shaper before the diffractive setup to efficiently illuminate the rectangular shaped spatial light modulator by a Gaussian laser beam. The method is initially tested for a single WOW and we have experimentally demonstrated dynamic tracking and coupling for both lateral and axial displacements of the WOWs. The ability to switch from on-demand to continuous addressing with efficient illumination leverages our WOWs for potential applications in near-field stimulation and nonlinear optics at small scales.
\end{abstract}

Keywords: Microfabrication, optical trapping, object tracking, diffractive optics, Generalized Phase Contrast

\section{INTRODUCTION}

Trapping and manipulation using light has progressed from a single tightly focused beam on a bead to orchestrated movements of multiple objects or even advanced multi-handled micro-tools ${ }^{1,2}$. Trapped objects are no longer restricted to a simple polystyrene bead but now extend to advanced and structured objects that can be readily fabricated using twophoton fabrication (2PP) $)^{3}$. Using 2PP fabrication allows full 3D flexibility in the design of desired structures so that a variety of forms and tasks can be carried out. An example is the optically-actuated surface scanning probe for investigating surface topography ${ }^{4}$. Instead of tightly focused traps, more softly focussed counter-propagating (CP) beams can also be used in multi-beam trapping. We have demonstrated this using polystyrene beads ${ }^{5}$ and also with fabricated extended objects that were used for demonstrating real-time optical microassembly ${ }^{6,7}$. The main advantage of using CP beams is the use of low-NA objective lenses to relay the trapping beams to the sample volume. The large working distance of low-NA objectives allows the possibility of adding side-view imaging of the sample as we have previously integrated on our proprietary Biophotonics Workstation (BWS ${ }^{8}$. In our recent work, we have fabricated free-floating waveguides that can be real-time optically manipulated in a volume coined Wave-guided Optical Waveguides (WOWs). The WOWs can serve as structure-mediated tools for redirecting light ${ }^{10}$ and for accessing targeted light delivery in difficult geometries. To allow dynamic coupling of light through the WOWs, we have added a diffractive setup that can independently modulate the coupling beams on our BWS ${ }^{11}$. Here, further improvements have been integrated by adding object tracking routines that allow real-time coupling to the WOWs while they are real-time manipulated in a volume. This leverages the capabilities of the WOWs for potential applications in near-field photo-stimulation and nonlinear optics at small scales. 


\section{Wave-guided Optical Waveguides}

Utilizing spherical handles the WOWs are conveniently trapped and manipulated by counter-propagating (CP) beams at a near-infrared wavelength. Lateral displacements of the WOWs are accomplished by simply dragging the CP-beams along the lateral direction while axial movements are performed by adjusting the intensity ratio of the respective CPbeams. As the WOWs are moved in 3D space, we require the coupling beams (green laser light) to real-time follow them for continuous addressing. The lateral displacements $\Delta x^{\prime}$ and $\Delta y^{\prime}$ of the WOWs can be readily obtained from the trapping interface since the CP-beams use an imaging geometry and thus only a simple scaling is needed for the hologram calculation. Since we have no direct access to the axial coordinate due to the nature of the trapping beams, we use an object tracking routine on the side-view imaging to automatically obtain the axial displacement coordinate shift $\Delta z^{\prime}$. A fluorescence dye is added to the trapping medium to help in visualizing the coupling beams using the side-view imaging on the BWS. The procedure is summarized by the schematic diagram and setup in Fig. 1:

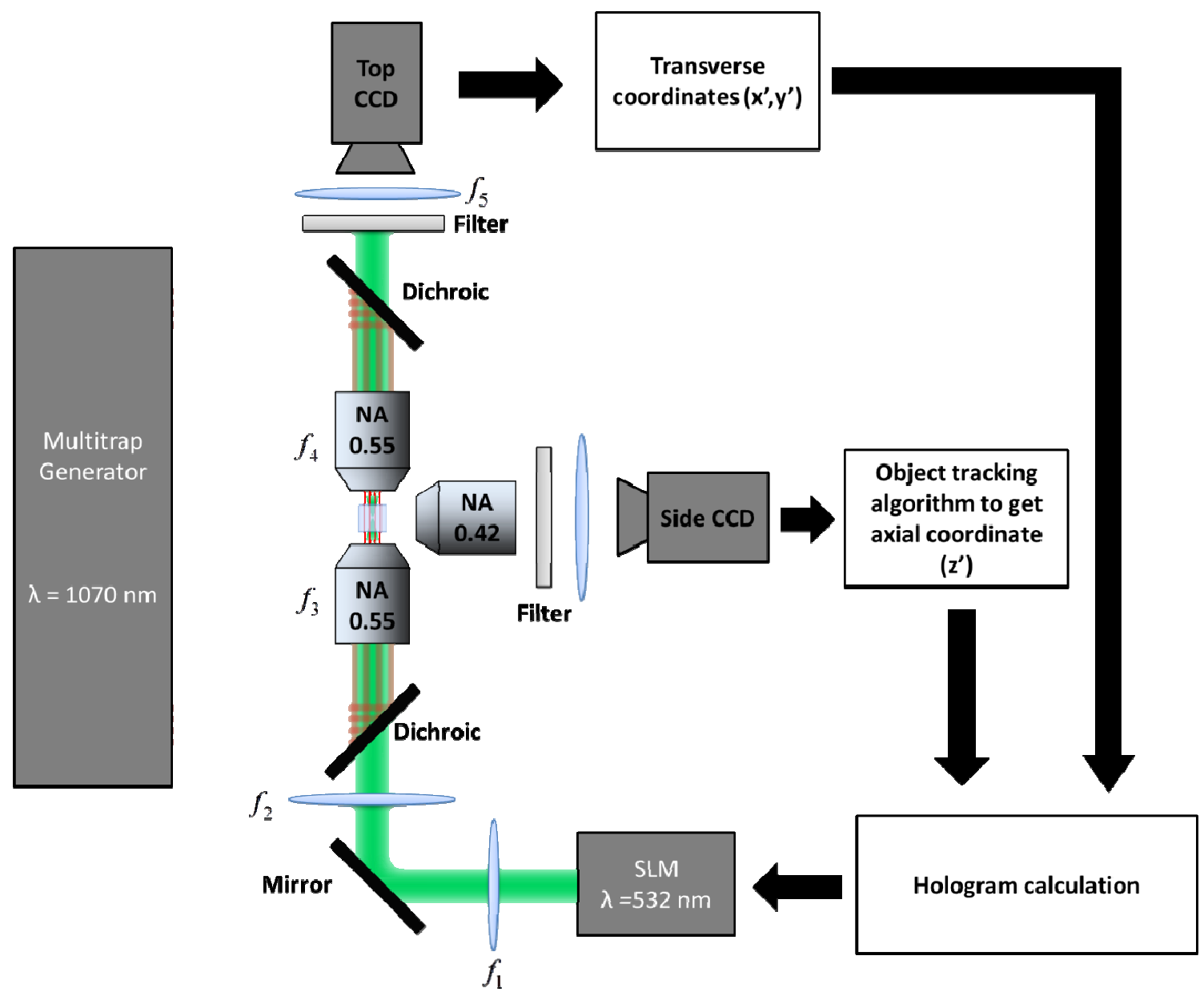

Figure 1. Schematic of the real-time diffractive coupling of the optically manipulated WOWs. The coordinates of each trapped WOW are obtained automatically for the calculation of the needed SLM-based real-time holograms.

The required real-time holograms for the lateral and axial displacements of the coupling beams are calculated using the fast but approximate so-called "prisms (blazed gratings) and lenses" approach given below:

$$
\phi_{\text {lateral }}(x, y)=\frac{2 \pi}{\lambda f}\left(x \Delta x^{\prime}+y \Delta y^{\prime}\right)
$$




$$
\phi_{\text {axial }}(x, y)=-\frac{\pi \Delta z^{\prime}}{\lambda f}\left(x^{2}+y^{2}\right)
$$

where $f$ is the focal length of the Fourier transforming lens. Occasionally, an offset is necessary to obtain convenient coordinates for both the trapping and the coupling beams. Thus the effective phase for a coupling beam is given by:

$$
\phi_{\text {eff }}(x, y)=\bmod \left(\phi_{\text {offset }}+\phi_{\text {lateral }}+\phi_{\text {axial }}, 2 \pi\right)
$$

The diffractive addressing of a single WOW can be visualized as shown in Fig.2 below:

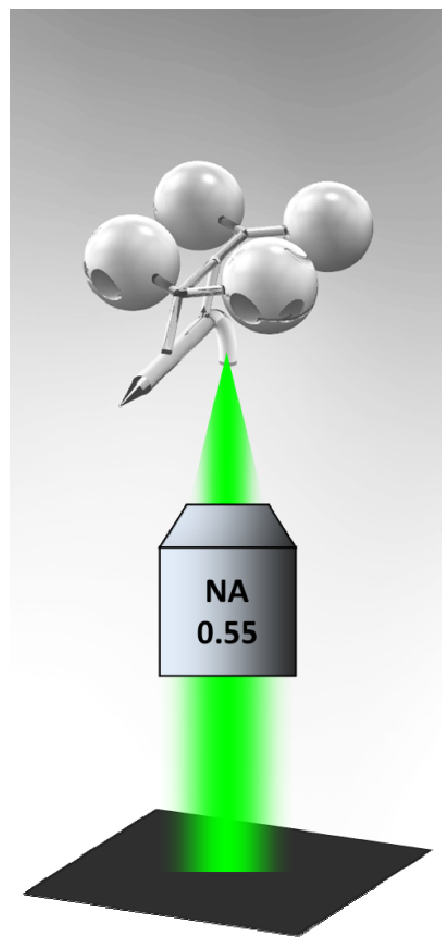

unmodulated

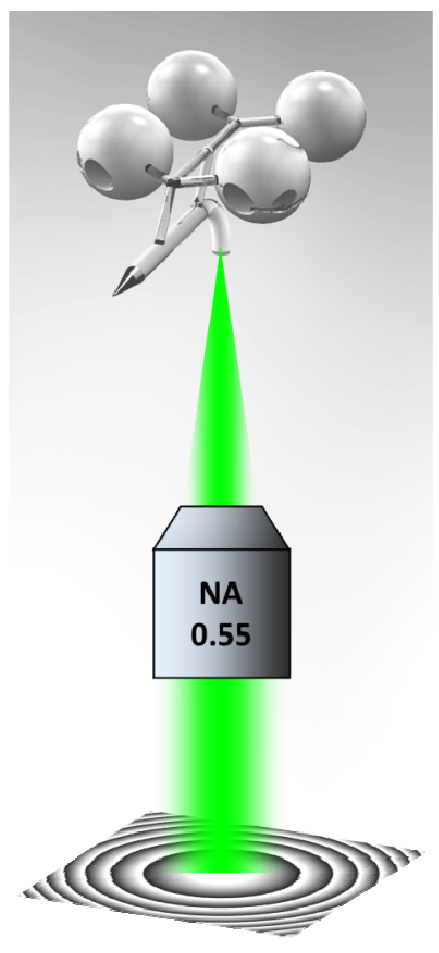

lens phase

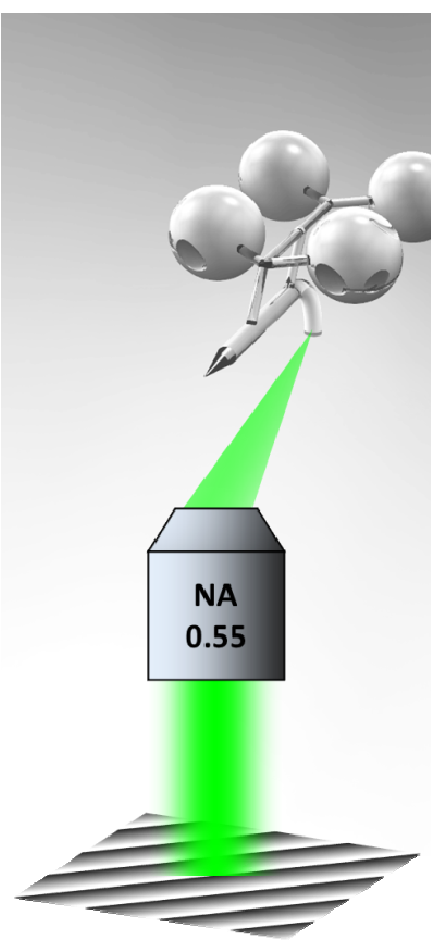

blazed grating phase

Figure 2. Graphical demonstration of holographic addressing of a single WOW. The WOW is moved in 3D space using the counter-propagating beams holding the four spherical handles of each WOW. The holograms are used to modulate the coupling beams to dynamically follow the position of each WOW in real-time.

To obtain an increased light efficiency of the coupling beams, a first static beam shaping using the Generalized Phase Contrast (GPC) method ${ }^{12}$ is performed to match the rectangular shape of the used SLM by an incident Gaussian laser beam profile. A compact module containing the main components of GPC is added to the diffractive setup as shown in Fig. 3. We refer to this module as the so-called GPC Light Shaper (LS). The size of the phase mask and Phase Contrast Filter (PCF) is optimized according to the values in our previous literature ${ }^{13}$. The use of the GPC LS allows for an efficient photon management compared to the more common practice of amplitude masking ${ }^{13,14}$.

The GPC LS works by introducing a phase shift to the incident Gaussian beam using an input phase mask that has a phase shifting region corresponding to the shape of the SLM (Hamamatsu Photonics, $798 \times 600$ pixels, $9.9 \mathrm{~mm} \times 7.5$ $\mathrm{mm}$ active area). A lens takes the Fourier transform of the phase shifted field and transforms it to the plane of the PCF which creates a so-called synthetic reference wave from the low spatial frequencies. A second lens takes another Fourier 
transform and the resulting pattern at the output is a contiguous intensity distribution corresponding one-to-one to the input phase mask. Unlike the common method of hard-truncation where photons are discarded, the GPC LS redirects photons to significantly enhance the photon-flux onto the SLM addressing surface where the coupling holograms are displayed.

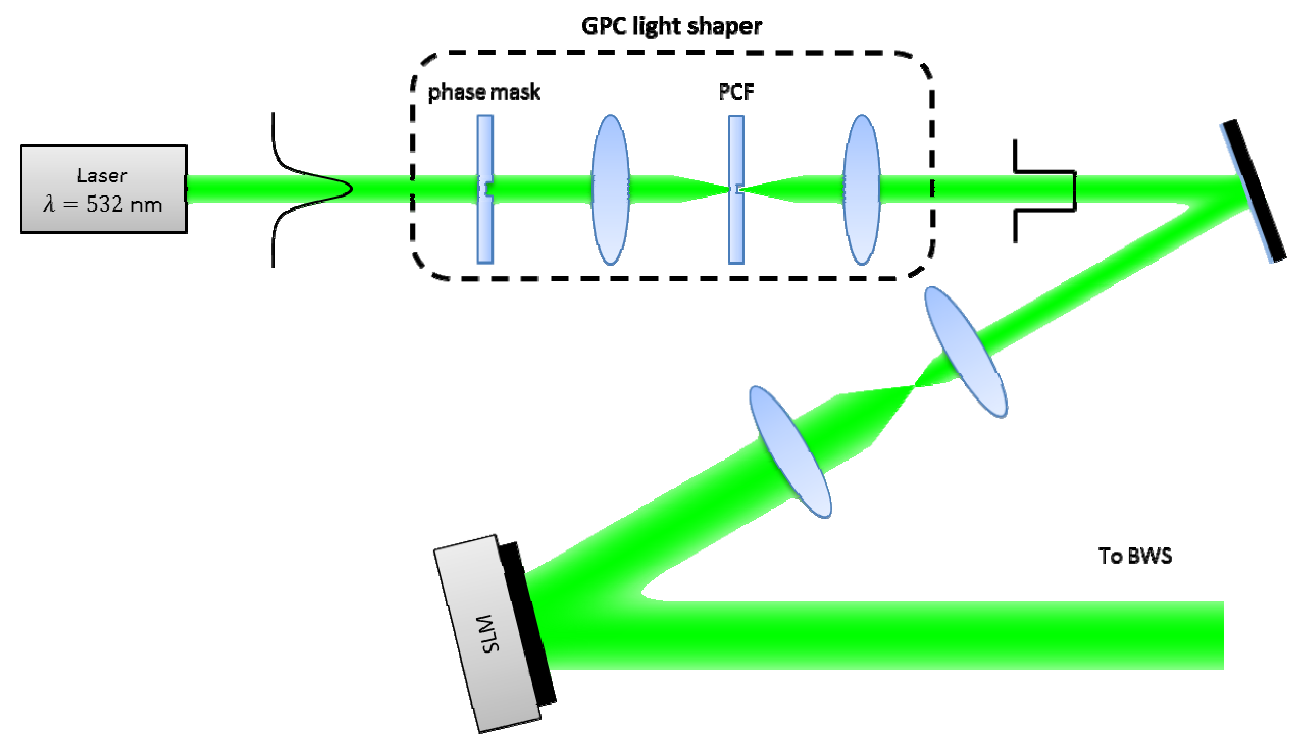

Figure 3. Schematic diagram of the real-time diffractive setup. The GPC LS is placed before the SLM to perform a static beam shaping to match the rectangular shape of the applied SLM. Unlike hard-truncation where a significant part of the photons are discarded, the GPC LS redirects the photons to where they are needed, on the SLM surface to obtain an enhanced read-out of the coupling holograms.

\section{RESULTS}

We applied a random arrangement of 20 - 40 focal spots for testing the SLM-based diffractive coupling to the WOWs (cf. Fig. 4). Our measurements presented in Fig. 5 shows a $\sim 3 \mathrm{x}$ gain when the GPC Light Shaper is used together with the diffractive setup compared with the usual hard-truncated Gaussian beam approach. The gain is calculated as the ratio of the average light spot intensity in the GPC-enhanced patterns to the average light spot intensity of the corresponding hard-truncated patterns. Qualitatively, the GPC-enhanced focal spots are brighter than their hard-truncated counterparts. As an example, the spots in a "40 spot-pattern" are still brighter compared to the spots in the hard-truncated case for a " 20 spot-pattern". This suggests that we can generate more intense focal spots that can be used for addressing a larger number of WOWs in addition to other advanced and simultaneous light excitations working in conjuction with these tools.

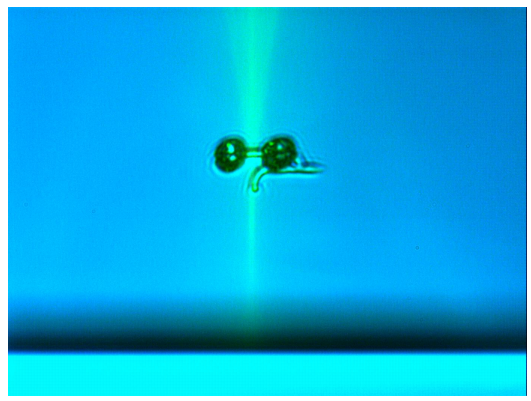

Figure 4. Side-view microscope image showing the experimental visualization of the focused light coupling to a single free-standing and optically trapped WOW. Here the trapped WOW is brought to the focus of a static green 
laser beam for effective light coupling. Subsequently, the green coupling-light is diffractively generated from a phase-only SLM to enable dynamic addressing and full 3D targeted light delivery for each WOW while they are being 3D manipulated in a volume. Adapted from ref. [11]
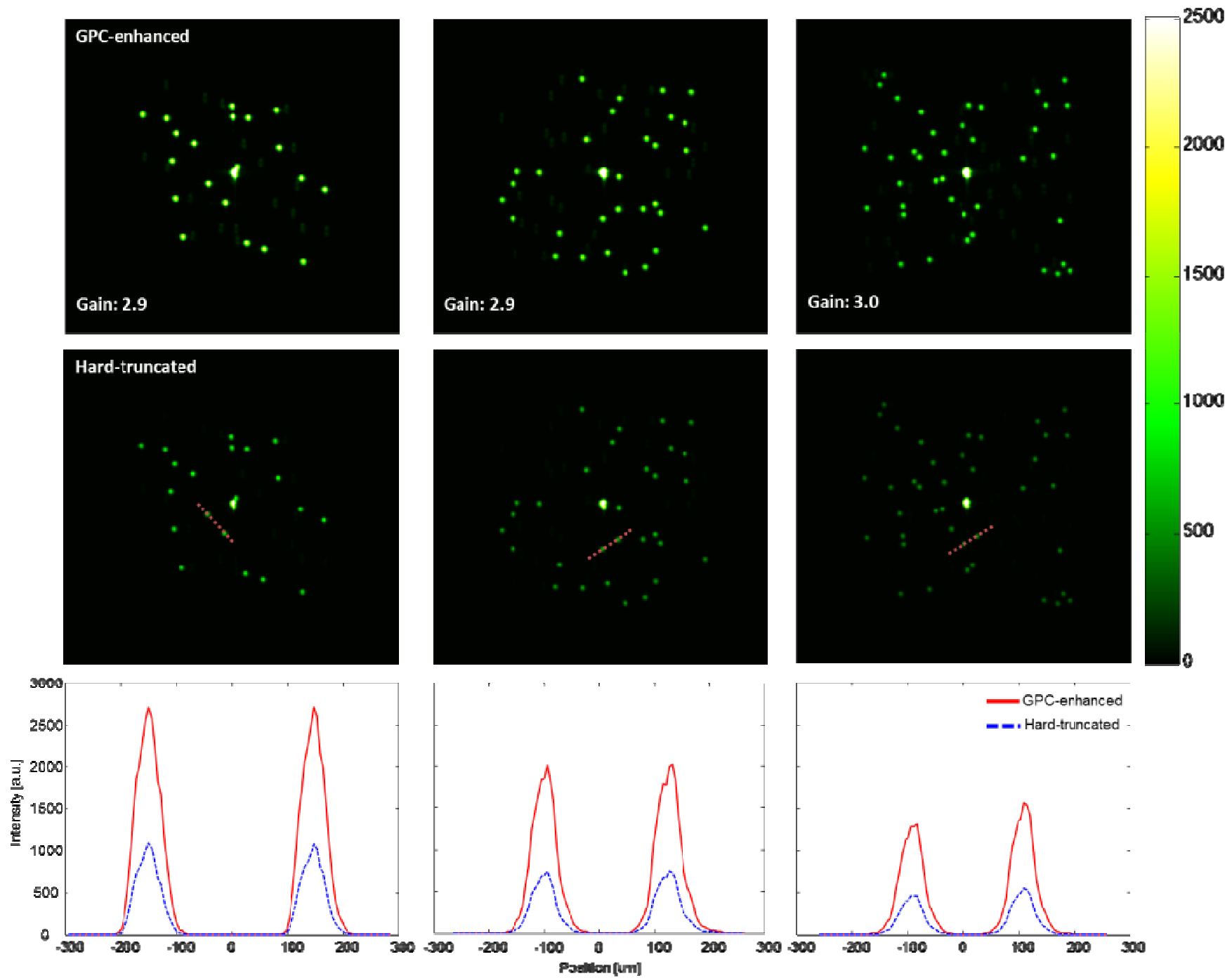

Figure 5. Experimental results showing that GPC-enhanced focal spots can be obtained with a $\sim 3 \mathrm{x}$ brighter intensity (red profiles) than their hard-truncated counterparts (blue profiles).

Real-time coupling has been tested for lateral and axial displacements of the WOWs, separately. Figs. 6 and 7 show snapshots from experiments performing real-time coupling during WOW-motion. Lateral displacement of a WOW is done by dragging the respective CP-traps on the BWS using a specially designed computer interface. The coordinate variables are then grabbed to calculate the required grating phase for the diffractive SLM-configuration. For axial addressing, as shown in Fig. 2b, the position of each WOW is obtained from a built-in object tracking routine implemented on LabVIEW. The obtained axial displacement is then used to calculate the required quadratic (lens) phase. The axial shifting of the coupling spots can be clearly observed from the fluorescence of the trapping medium. The use of our proprietary GPC Light Shaper prior to the SLM phase modulation produces significantly more intense coupling spots that are desirable for addressing a plurality of WOWs simultaneously. The GPC Light Shaper can be implemented 
using a fixed phase filter ${ }^{15}$, using a more advanced self-induced ${ }^{16}$ approach or by implementing a self-aligned phase filter ${ }^{17}$ approach.

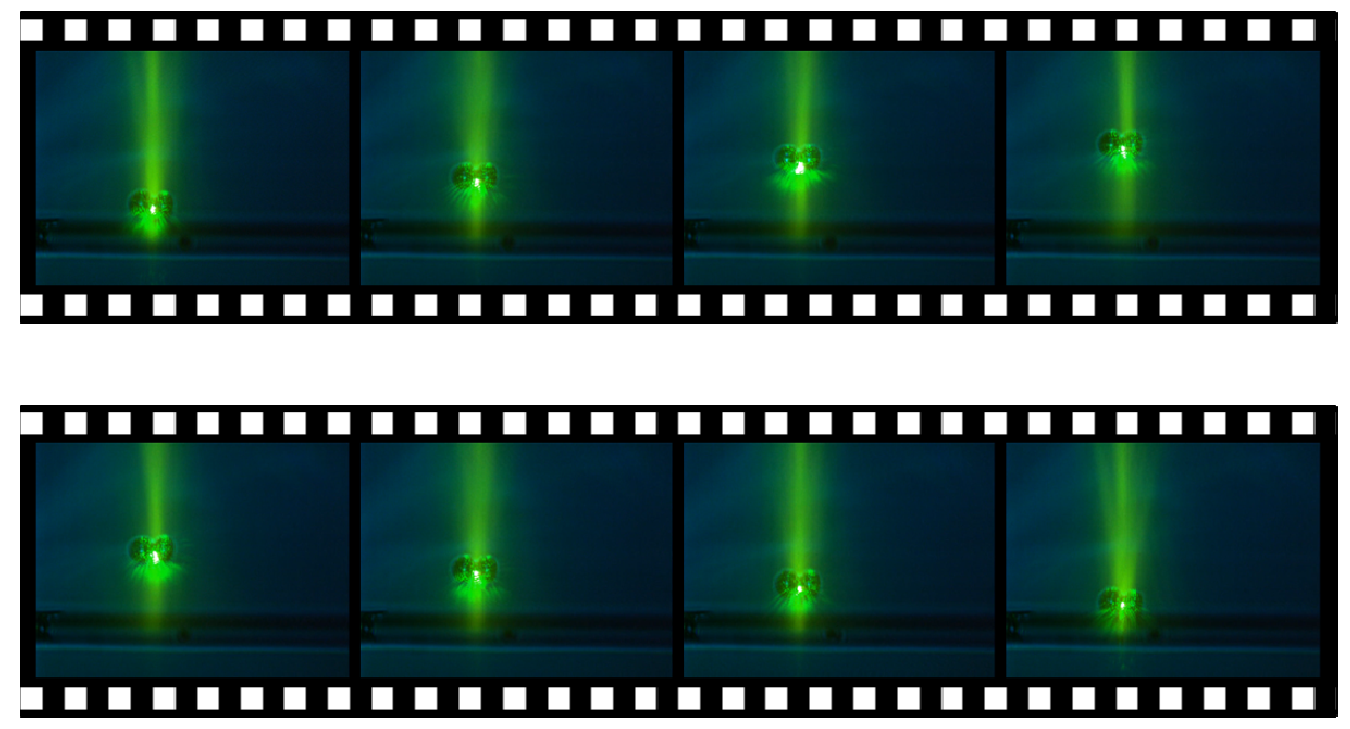

Figure 6. Dynamic experimental snapshots demonstrating real-time WOW-coupling along the axial direction.
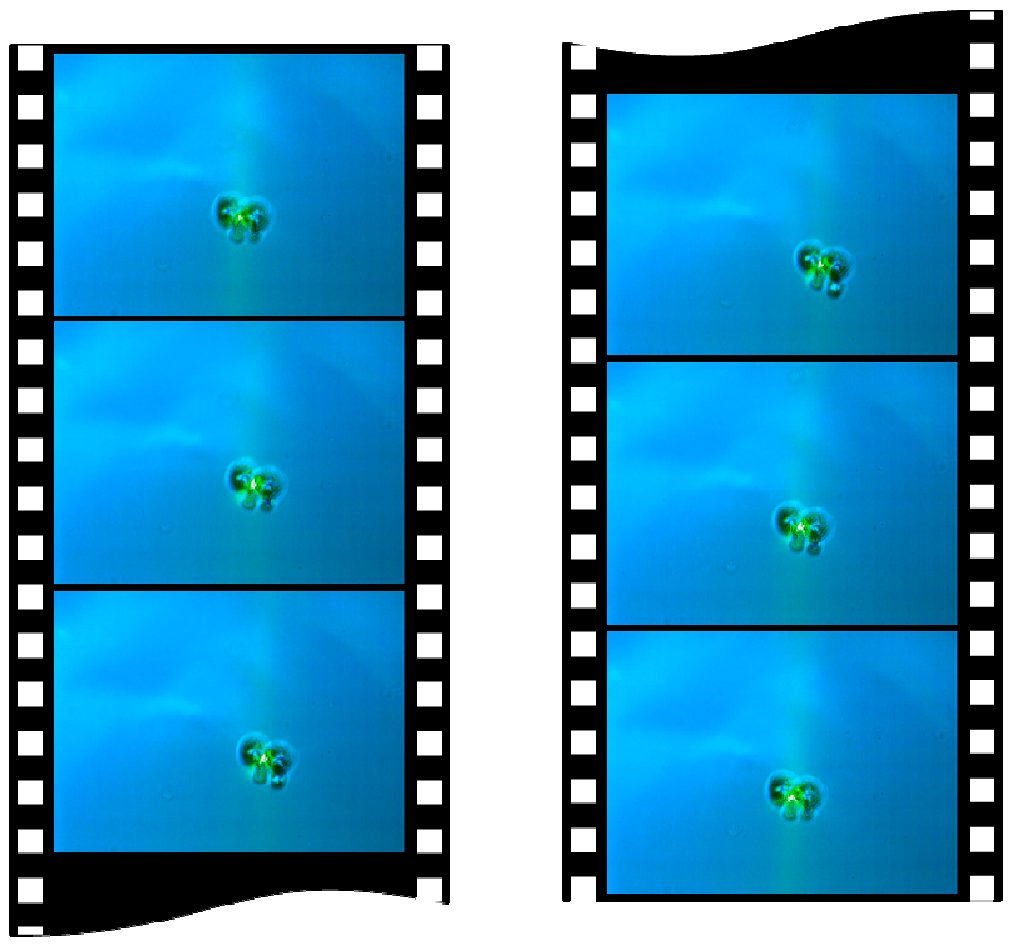

Figure 7. Dynamic experimental snapshots demonstrating real-time WOW-coupling along the lateral direction. 


\section{CONCLUSIONS}

This work experimentally demonstrate dynamic and reconfigurable coupling of green laser light to so-called Waveguided Optical Waveguides (WOWs) by using an advanced real-time object tracking algorithm. The ability to switch between on-demand and continuous coupling offers a versatile approach for the WOWs for potential applications in near-field photo-stimulation and nonlinear optics at small scales. The addition of a proprietary GPC Light Shaper in the SLM-based diffractive setup allows efficient formation of high intensity light spot patterns that are desirable when addressing a plurality of real-time manipulated WOWs in a volume.

\section{ACKNOWLEDGMENTS}

This work is supported by Enhanced Spatial Light Control in Advanced Optical Fibres (e-space), a project financed by the Innovation Fund Denmark. We acknowledge the support from Hamamatsu Photonics.

\section{REFERENCES}

[1] Ashkin, A., “Acceleration and Trapping of Particles by Radiation Pressure,” Phys. Rev. Lett. 24(4), 24-27 (1970).

[2] Glückstad, J., "Optical manipulation: Sculpting the object," Nature Photonics, Vol. 5, 7-8 (2011)

[3] Palima, D ., Glückstad, J., "Gearing up for optical microrobotics: micromanipulation and actuation of synthetic microstructures by optical forces," Laser Photon. Rev. 7(4), 478-494 (2013).

[4] Phillips, D. B., Gibson, G. M., Bowman, R., Padgett, M. J., Hanna, S., Carberry, D. M., Miles, M. J ., Simpson, S. H., "An optically actuated surface scanning probe," Opt. Express 20(28), 29679-29693 (2012).

[5] Rodrigo, P. J., Perch-Nielsen, I. R., Alonzo, C. A ., Glückstad, J., "GPC-based optical micromanipulation in 3D real-time using a single spatial light modulator.," Opt. Express 14(26), 13107-13112 (2006).

[6] Rodrigo, P. J., Gammelgaard, L., Bøggild, P., Perch-Nielsen, I ., Glückstad, J., “Actuation of microfabricated tools using multiple GPC-based counterpropagating-beam traps," Opt. Express 13(18), 6899-6904 (2005).

[7] Rodrigo, P. J., Kelemen, L., Palima, D., Alonzo, C. A., Ormos, P ., Glückstad, J., "Optical microassembly platform for constructing reconfigurable microenvironments for biomedical studies.," Opt. Express 17(8), 65786583 (2009).

[8] Ulriksen, H.-U., Thøgersen, J., Keiding, S., Perch-Nielsen, I. R., Dam, J. S., Palima, D. Z., Stapelfeldt, H ., Glückstad, J., "Independent trapping, manipulation and characterization by an all-optical biophotonics workstation,” J. Eur. Opt. Soc. Rapid Publ. 3, 08034 (2008).

[9] Palima, D., Bañas, A., Vizsnyiczai, G., Kelemen, L., Ormos, P ., Glückstad, J., "Wave-guided optical waveguides," Opt. Express 20(3), 2004-2014 (2012).

[10] Glückstad, J., Bañas, A., Aabo, T ., Palima, D., "Structure-mediated micro-to-nano coupling using sculpted light and matter," Proc. SPIE Vol. 8424 8424, D. L. Andrews, J.-M. Nunzi, and A. Ostendorf, Eds. (2012).

[11] Villangca, M., Bañas, A., Palima, D ., Glückstad, J., "Dynamic diffraction-limited light-coupling of 3Dmaneuvered wave-guided optical waveguides," Opt. Express 22(15), 17880-17889 (2014).

[12] Glückstad, J ., Palima, D., "Generalized Phase Contrast: Applications in Optics and Photonics," Springer Series in Optical Sciences, Vol. 146, 1-311 (2009).

[13] Bañas, A., Palima, D., Villangca, M., Aabo, T ., Glückstad, J., "GPC light shaper for speckle-free one-and twophoton contiguous pattern excitation," Opt. Express 22(5), 5299-5310 (2014).

[14] Bañas, A., Kopylov, O., Villangca, M., Palima, D ., Glückstad, J., "GPC light shaper: static and dynamic experimental demonstrations.," Opt. Express 22(20), 23759-23769 (2014).

[15] Glückstad, J., Mogensen, P.,"Reconfigurable ternary-phase array illuminator based on the generalised phase contrast method," Opt. Commun. 173, 169-175 (2000).

[16] Glückstad, J., "Adaptive array illumination and structured light generated by spatial zero-order self-phase modulation in a Kerr medium," Opt. Commun. 120, 194-203 (1995).

[17] Kenny, F., Choi, F., Glückstad, J., Booth, M., "Adaptive optimisation of a generalised phase contrast beam shaping system," Optics Communications 342, 109-114 (2015). 